\title{
A Practical Visual Servo Control for an Unmanned Aerial Vehicle
}

\author{
Nicolas Guenard, Tarek Hamel, Member, IEEE, and Robert Mahony, Senior Member, IEEE
}

\begin{abstract}
An image-based visual servo control is presented for an unmanned aerial vehicle (UAV) capable of stationary or quasistationary flight with the camera mounted onboard the vehicle. The target considered consists of a finite set of stationary and disjoint points lying in a plane. Control of the position and orientation dynamics is decoupled using a visual error based on spherical centroid data, along with estimations of the linear velocity and the gravitational inertial direction extracted from image features and an embedded inertial measurement unit. The visual error used compensates for poor conditioning of the image Jacobian matrix by introducing a nonhomogeneous gain term adapted to the visual sensitivity of the error measurements. A nonlinear controller, that ensures exponential convergence of the system considered, is derived for the full dynamics of the system using control Lyapunov function design techniques. Experimental results on a quadrotor UAV, developed by the French Atomic Energy Commission, demonstrate the robustness and performance of the proposed control strategy.
\end{abstract}

Index Terms-Aerial robotic vehicle, experiments, image-based visual servo (IBVS), underactuated systems.

\section{INTRODUCTION}

V ISUAL servo algorithms have been extensively developed in the robotics field over the last 10 years [11], [30]. Visual servo systems may be divided into two main classes [24]; posebased visual servo (PBVS) control or image-based visual servo (IBVS) control. Position-based visual servo control (PBVS) involves reconstruction of the target pose with respect to the robot

Manuscript received September 21, 2006; revised June 2, 2007. This paper was recommended for publication by Associate Editor P. Rives and Editor K. Lynch upon evaluation of the reviewers' comments. This work was supported by the French company Wany Robotics (www.wanyrobotics.com) under a CEA-Wany Ph.D. Grant and a CNRS PICS Grant (France-Australia).

N. Guenard is with the Laboratoire d'Intégration des Systèmes et des Technologies (CEA/LIST), French Atomic Energy Commission, 92265 Fontenayaux-Roses Cedex, France (e-mail: guenardn@zoe.cea.fr).

T. Hamel is with the Laboratoire I3S, University of Nice-Sophia AntipolisNational Centre for Scientific Research (UNSA-CNRS), 06108 Nice Cedex, France (e-mail: thamel@i3s.unice.fr).

R. Mahony is with the Department of Engineering, Australian National University, Canberra, A.C.T. 0200, Australia (e-mail: robert.mahony@anu.edu.au).

This paper has supplementary downloadable multimedia material available at http://ieeexplore.ieee.org. provided by the author. This material includes one video (ReadMe) demonstrating a target consisting of four black marks on the vertices of a stationary planar square. A standard computer vision segmentation algorithm extracts the marks from the background and computes the central moment of each mark. The central moments are transformed into unit-norm spherical image plane representation using the camera calibration matrix provided by the manufacturer. The experiments show that the regulation error remains bounded and smaller than $10 \mathrm{~cm}$ around the desired position. Practical stability is very good with regard to the experimental system considered. The robustness of the control scheme is demonstrated by disturbing the system. The size of the video is not available. Contact thamel@i3s.unice.fr for further questions about this work.

Color versions of one or more of the figures in this paper are available online at http://ieeexplore.ieee.org.

Digital Object Identifier 10.1109/TRO.2008.916666 and results in a Cartesian motion planning problem. This approach requires an accurate 3-D model of the target, is sensitive to camera calibration errors, and displays a tendency for image features to leave the camera field of view during the task evolution. Image-based visual servo control treats the problem as one of the controlling features in the image plan such that moving features to a goal configuration implicitly result in the task being accomplished [11]. Feature errors are mapped to actuator inputs via the inverse of an image Jacobian matrix. There are a wide range of features that have been considered including points, lines, circles, and image moments. Different features lead to different closed-loop responses and there has been important research into optimal selection of features and partitioned control where some degrees of freedom are controlled visually and others by a second sensor modality [5], [19]. Image-based visual servo control avoids many of the robustness and calibration problems associated with PBVS, however, it has its own problems [6]. Foremost in the classical approach is a requirement to estimate the depth of each feature point in the visual data. Various solutions have been investigated, including estimation via partial pose estimation [24], adaptive control [28], and estimation of the image Jacobian using quasi-Newton technics [29]. More recently, there has been considerable interest in hybrid control methods whereby translational and rotational control are treated separately [8], [10], [24], [27]. Most existing IBVS approaches were developed for serial-link robotic manipulators [18]. For this kind of robot, there are low-level joint controllers that compensate for system dynamics, and position control, such as visual servo control, is undertaken at the level of the system kinematics [11]. There are very few integrated IBVS control designs for fully dynamic system models [3], [34] and even fewer that deal with underactuated dynamic models such as unmanned aerial vehicles (UAVs) [14], [26]. The key challenge in applying classical visual servo control to a dynamic system model lies in the highly coupled form of the image Jacobian. Much of the existing research in visual servo control of aerial robots (and particularly, autonomous helicopters) have used PBVS methodology [1], [25], [31] that avoids the image Jacobian formulation. Prior research by the authors [14] proposed a theoretical IBVS control design for a class of underactuated dynamics using an image-based visual feature augmented with an inertial direction, obtained from a partial attitude pose-estimation algorithm. In [15], a fully IBVS control design for dynamic systems associated with UAV systems capable of hover fight, is derived. Both control schemes assume that the translational velocity of the system is measured directly. In [22], an IBVS control for a fully dynamic system is designed for a translational motion of a rigid body. The image features considered are a first-order 


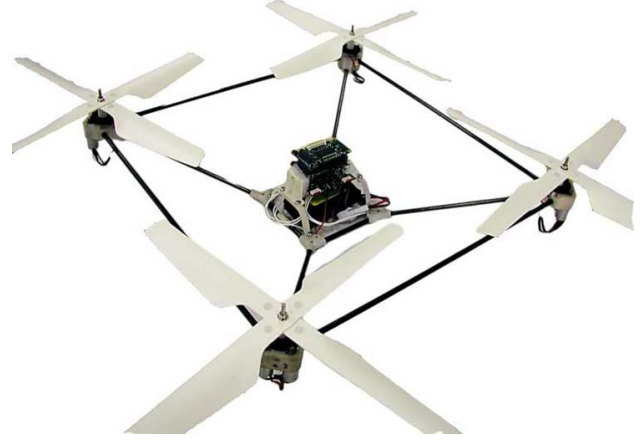

Fig. 1. X4-flyer UAV.

unnormalized spherical moment for position stabilization and optic flow for velocity. Direct implementation of the control strategies proposed in [14], [15] and [12] has been found to have poor sensitivity and conditioning when implemented directly on an experimental vehicle.

In this paper, the practical implementation of an IBVS control for an UAV, capable of stationary or quasi-stationary flight, is presented. The model considered is that of an "eye-in-hand" type configuration, where the camera is attached to the airframe of the UAV. The approach taken is based on recent research by the authors [14] for which the dynamics of the image features have certain passivity-like properties. A new visual error term is considered that improves the conditioning of the image Jacobian. The initial analysis is undertaken for the kinematic response of the system, the normal visual servo framework, and shows that the resulting image Jacobian is well conditioned. Following this, a nonlinear controller integrating the linear and rotational dynamics is developed using a structured control Lyapunov function for exponential stabilization of the full dynamics of the UAV. The vehicle considered is equipped with an inertial measurement unit (IMU) and an explicit complementary filter is used to provide filtered estimates of attitude [16] and angular velocity for the vehicle. An estimate of translational velocity is derived from a nonlinear filter that fuses the IMU and visual data [7]. Experimental results are obtained on a quadrotor UAV system, developed by the French Atomic Energy Commission (CEA), capable of stationary and quasi-stationary flight. The closed-loop visual servo control is shown to be locally exponentially stable and experimental results demonstrate the performance and robustness of the proposed control.

The paper is arranged into six sections. Following the introduction, Section II presents the fundamental equations of motion for a quadrotor UAV. Section III presents the proposed choice of image features. Section IV provides a kinematic control design for the translational motion. Section $\mathrm{V}$ extends the control to the full dynamics of the system. Section VI presents experimental results obtained on the experimental quadrotor (Fig. 1). Finally Section VII provides some concluding remarks.

\section{DYNAMIC MODEl FOR AN HOVERING UAV}

In this section, we present equations of motion for an UAV in quasi-stationary (or hover) flight conditions. The model used is based on those introduced in the literature to model the dynam- ics of helicopters [13], [17], [31]. Let $\mathcal{I}=\left\{E_{x}, E_{y}, E_{z}\right\}$ denote a right-hand inertial or world frame such that $E_{z}$ denotes the vertical direction downward into the earth. Let $\xi=(x, y, z)$ denote the position of the center of mass of the object in the inertial frame $\mathcal{I}$. Let $\mathcal{A}=\left\{E_{1}^{a}, E_{2}^{a}, E_{3}^{a}\right\}$ be a (right-hand) body-fixed frame centered at the center of mass and assume that it coincides with the camera frame. The orientation of the airframe is given by a rotation $R: \mathcal{A} \rightarrow \mathcal{I}$, where $R \in S O(3)$ is an orthogonal rotation matrix. Let $V \in \mathcal{A}$ denote the linear velocity and $\Omega \in \mathcal{A}$ denote the angular velocity of the camera both expressed in the camera frame. Let $m$ denote the mass of the rigid object and let $\mathbf{I} \in \mathbb{R}^{3 \times 3}$ be the constant inertia matrix around the center of mass (expressed in the body-fixed frame $\mathcal{A}$ ). The dynamics of a rigid body are

$$
\begin{aligned}
\dot{\xi} & =R V \\
m \dot{V} & =-m \Omega \times V+F \\
\dot{R} & =R \operatorname{sk}(\Omega) \\
\mathbf{I} \dot{\Omega} & =-\Omega \times \mathbf{I} \Omega+\Gamma .
\end{aligned}
$$

where the notation $\mathrm{sk}(x)$ denotes the skew-symmetric matrix associated with any vector $x \in \mathbb{R}^{3}$ such that for any vector $y \in \mathbb{R}^{3}, \operatorname{sk}(x) y=x \times y$.

The exogenous force and torque are denoted by $F$ and $\Gamma$, respectively. The inputs considered correspond to a typical arrangement found on a vertical take-off and landing (VTOL) aircraft (Section VI). The inputs are written as a single translational force, denoted $F$, along with full torque control, $\Gamma=\left(\Gamma_{1}, \Gamma_{2}, \Gamma_{3}\right)^{T}$ around axes $\left(E_{1}^{a}, E_{2}^{a}, E_{3}^{a}\right)$. The force $F$ combines thrust, lift, gravity, and drag components. It is convenient to separate the gravity component ${ }^{1} m g E_{z}=m g R^{T} e_{3}$ from the combined aerodynamic forces and assume that the aerodynamic forces are always aligned with the $z$-axis in the body-fixed frame

$$
F:=-T e_{3}+m g R^{T} e_{3}
$$

where $T \in \mathbb{R}$ is a scalar input representing the magnitude of the external force applied in direction $e_{3}$. This is a reasonable assumption for the dynamics of a UAV in quasi-stationary flight, where the exogenous force is dominated by the lift force while the aerodynamical drag (depending on the square of the linear velocity) and forward thrust are negligible [13], [22], [32]. Control of the airframe is obtained by using the torque control $\Gamma=\left(\Gamma_{1}, \Gamma_{2}, \Gamma_{3}\right)$ to align the force (or thrust vector) $F_{0}:=T E_{3}^{a}=T e_{3}$ as required to track the goal trajectory.

\section{Choice of Image Features}

\section{A. Kinematics of an Image Point Under Spherical Projection}

Let $P$ be a stationary point target visible to the camera expressed in the camera frame. The image point observed by the camera is denoted $p$ and is obtained by rescaling onto the image surface $\mathcal{S}$ of the camera. Following the approach introduced in [14], we consider a camera with a spherical image plane.

\footnotetext{
${ }^{1}$ Here $e_{3}=\left(\begin{array}{lll}0 & 0 & 1\end{array}\right)$ denotes the third-axis unit vector in $\mathbb{R}^{3}$.
} 
Thus

$$
p=\frac{P}{|P|}
$$

where $|x|$ represents the Euclidian norm of any vector $x \in \mathbb{R}^{n}$, $|x|=\sqrt{x^{T} x}$. The dynamics of an image point for a spherical camera of image surface radius unity are [4], [14]

$$
\dot{p}=-\Omega \times p-\frac{\pi_{p}}{r} V
$$

where $r=|P|$ and $\pi_{p}=\left(I_{3}-p p^{T}\right)$ is the projection $\pi_{p}$ : $\mathbb{R}^{3} \rightarrow T_{p} \mathcal{S}^{2}$, onto the tangent space of the sphere $S^{2}$ at the point $p \in \mathcal{S}^{2}$.

\section{B. Centroid of a Target Surface}

Consider a point target consisting of $N$ points $\left\{P_{i}\right\}$ with image points $\left\{p_{i}\right\}$. The centroid of a point target is defined to be

$$
q_{0}:=\frac{\sum_{i=1}^{N} p_{i}}{\left|\sum_{i=1}^{N} p_{i}\right|} \in \mathcal{S}^{2} .
$$

The centroid measures the center of mass of the observed points in the chosen camera geometry. The centroid depends implicitly on the camera geometry, and for a different geometry (such as a camera with perspective projection), the direction of the centroid will be different.

Using the centroid information is an old technique in visual servo control [2], [20], [33]. Among the advantages in computing target centroids; it is not necessary to match the observed image points with desired features as would be necessary in a classical image-based visual servo control [18], the calculation of an image centroid is highly robust to pixel noise, and centroids are easily computed in real time. The disadvantage of the definition (8) is that it measures only two degrees of freedom associated with the direction of centroid with respect to the body-fixed-frame axes of the camera. The unnormalized spherical centroid is defined to be

$$
q:=\sum_{i=1}^{N} p_{i} \in \mathbb{R}^{3} .
$$

Intuitively, as the camera approaches the geometric center of the target points for a spherical camera geometry, the observed image points spread out around the focal point of the camera, decreasing the norm of $q$. In the limit, the value of $q$ can theoretically reach zero, although for most practical systems, this will not be possible while keeping the image points in the field of view of the camera. Conversely, as the camera moves away from the geometric center of the target points, the observed image points cluster together in the direction of the image. The unnormalized centroid $q$ converges to a vector that has norm $N$ and points toward the target. This relationship between the norm of $q$ and the distance to the target provides a third constraint in the image-based error term. It is, however, highly nonlinear and leads to sensitivity and conditioning problems that must be overcome in the control design.
For a point target comprising a finite number of image points, the kinematics of the image centroid are easily verified to be

$$
\dot{q}=-\Omega \times q-Q V
$$

where

$$
Q=\sum_{i=1}^{i=n} \frac{\pi_{p_{i}}}{\left|P_{i}\right|}
$$

is a positive definite matrix as long as there are at least two $(N \geq 2)$ visible target points [14].

\section{Image-Based Errors}

In this paper, we augment the image information with inertial information acquired from a standard IMU used in most smallscale UAVs.

Formally, let $b \in \mathcal{I}$ denote the desired inertial direction for the visual feature. The norm of $b$ encodes the effective depth information for the desired limit point. Define

$$
q^{*}:=R^{T} b \in \mathcal{A}
$$

to be the desired target vector expressed in the camera-fixed frame. The orientation matrix $R$ is estimated from filtered data acquired on a strapdown IMU on the vehicle. Since $q^{*} \in \mathcal{A}$, it inherits dynamics from the motion of the camera

$$
\dot{q}^{*}=-\Omega \times q^{*} .
$$

The natural image-based error is the difference between the measured centroid and the target vector expressed in the camera frame

$$
\delta:=q-q^{*} .
$$

The image error kinematics are

$$
\dot{\delta}=-\Omega \times \delta-Q V .
$$

To regulate the full pose of the camera using a fully actuated kinematic system (such as a robotic manipulator), it would be necessary to introduce an additional error criterion for orientation control.

For an underactuated dynamic system of the form 1-4, the attitude dynamics are used to control the orientation of the vehicle thrust, which, in turn, provides the control of the system position dynamics. It is physically impossible to separately stabilize the attitude and position of the camera. The error criterion chosen regulates only the position of the rigid body and the orientation regulation is derived as a consequence of the system dynamics.

\section{Kinematic Control Design}

In this section, a Lyapunov control design is given for the kinematics of the translational motion (1) based on the visual error (13).

Define a storage function $S$

$$
S=\frac{1}{2}|\delta|^{2} .
$$


Taking the time derivative of $S$ and substituting for (13) yields

$$
\dot{S}=-\delta^{T} Q V
$$

Note that (15) is independent of the angular velocity $\Omega$.

For $N \geq 2$, the matrix $Q>0$ is known to be positive definite, and although its exact structure is not known, its maximal eigenvalue must satisfy

$$
\lambda_{\max }(Q) \leq \sum_{i=1}^{i=n} \frac{1}{\left|P_{i}\right|}
$$

where $\left|P_{i}\right|$ denotes the relative depth of the $i$ th image point. Thus, a simple choice

$$
V=k_{\delta} \delta, \quad k_{\delta}>0
$$

is sufficient to stabilize $S$ for a kinematic control regime. Indeed, substituting into (15), one obtains

$$
\dot{S}=-k_{\delta} \delta^{T} Q \delta
$$

Since $Q$ is a positive-definite matrix, the classical Lyapunov theory guarantees that $\delta$ converges exponentially to zero. The eigenvalues of the matrix $Q$ are generally ill-conditioned

$$
\lambda_{\min }(Q)<<\lambda_{\max }(Q) .
$$

Convergence rates of the components of the error $\delta$ depend on the eigenvalues of $Q$. As a consequence, the natural control $V=k_{\delta} \delta$ leads to poor asymptotic performance of the closedloop system.

\section{A. Compensation of the Control Gain Sensitivity}

A number of different approaches have been proposed to compensate the poor conditioning of the Jacobian matrix $Q$ and to improve the performance of the closed-loop system [4]. In earlier research, only the kinematic model was studied and the dynamics of the system were not considered in the control design. In this paper, we propose a modification of the visual error term to improve the conditioning of the Jacobian matrix $Q$ in the neighborhood of the set point $q^{*}$, thus preserving the passivity-like properties and allowing control design for the full dynamics of the system.

At the set point, the Jacobian matrix $Q$ displays two eigenvalues of comparable magnitude and one eigenvalue, associated with the direction $q^{*}$, which is an order of magnitude smaller. To deal with this ill-conditioning, two new error terms are introduced

$$
\delta_{11}=q_{0}^{*} \times q, \quad \delta_{12}=q_{0}^{* T} \delta, \quad q_{0}^{*}=\frac{q^{*}}{\left|q^{*}\right|} .
$$

Differentiating $\delta_{11}$ and $\delta_{12}$, it follows that

$$
\begin{aligned}
& \dot{\delta}_{11}=-\operatorname{sk}(\Omega) \delta_{1}-\operatorname{sk}\left(q_{0}^{*}\right) Q V \\
& \dot{\delta}_{12}=-q_{0}^{* T} Q V .
\end{aligned}
$$

Lemma 4.1 Consider the system defined by (13) and let $k_{1}, \lambda>0$ be two strictly positive constants. Define

$$
\delta_{1}=\delta_{11}+\lambda q_{0}^{*} \delta_{12}
$$

Assume that the image remains in the camera field of view for all time. Then, the closed-loop system (13) based on the following control (21)

$$
V=k_{1}\left(-\operatorname{sk}\left(q_{0}^{*}\right)+\lambda q_{0}^{*} q_{0}^{* T}\right)\left(\delta_{11}+\lambda q_{0}^{*} \delta_{12}\right)
$$

exponentially stabilizes the visual error $\delta_{1}$, and therefore, $\delta$.

Proof:

Define

$$
S_{1}=\frac{1}{2}\left|\delta_{11}\right|^{2}+\lambda^{2}\left|\delta_{12}\right|^{2} .
$$

It is straightforward to verify that the two components of $\delta_{1}\left(\delta_{11}\right.$ and $q_{0}^{*} \delta_{12}$ ) are orthogonal, and therefore,

$$
S_{1}=\frac{1}{2}\left|\delta_{11}+\lambda q_{0}^{*} \delta_{12}\right|^{2}=\frac{1}{2} \delta_{1}^{2} .
$$

Deriving $S_{1}$ and substituting the control input $V$ by its expression, yields

$$
\dot{S}_{1}=-k_{1} \delta_{1}^{T} H \delta_{1}
$$

where

$$
H=A\left(q_{0}^{*}\right) Q A\left(q_{0}^{*}\right)^{T}, \quad A\left(q_{0}^{*}\right)=-\operatorname{sk}\left(q_{0}^{*}\right)+\lambda q_{0}^{*} q_{0}^{* T} .
$$

Since $Q$ is positive-definite matrix and $A\left(q_{0}^{*}\right)$ is a nonsingular matrix, $H>0$, and therefore, $\delta_{1}$ (respectively $\delta$ ) converges exponentially to zero.

The decoupling between $\delta_{11}$ and $\delta_{12}$ and the decrease of the storage function $S_{1}$ toward zero guarantee the exponential convergence of the error $\delta$ to zero.

Remark 4.2: The best choice of the gain $\lambda$ is characterized by setting

$$
H \cong I
$$

where the symbol $\cong$ means "equality up to a multiplicative constant." Although this relationship cannot be exactly assigned, it can be approximately satisfied over a large neighborhood around the desired set point and overcomes the inherent sensitivity and conditioning of the control law proposed in [14].

\section{CONTROl Design FOR THE Full DynAmics}

In this section, the kinematic control developed in Section IV is adapted to apply to the full underactuated system dynamics using the backstepping control Lyapunov function design approach.

The dynamics of the error term $\delta_{1}(20)$ may be written

$$
\dot{\delta}_{1}=-\mathrm{sk}(\Omega) \delta_{1}-\frac{k_{1}}{m} H \delta_{1}-\frac{k_{1}}{m} H \delta_{2}
$$

where $\delta_{2}$ defines the difference between the desired (or virtual) kinematic controller (21) and the true velocity

$$
\delta_{2}:=\frac{m}{k_{1}} A\left(q_{0}^{*}\right)^{-T} V-\delta_{1}
$$


and will form an error term to stabilize the translational dynamics. With the aforementioned definitions, one has

$$
\dot{S}_{1}=-\frac{k_{1}}{m} \delta_{1}^{T} H \delta_{1}-\frac{k_{1}}{m} \delta_{1}^{T} H \delta_{2} .
$$

It is easily verified that

$$
\left(A\left(q_{0}^{*}\right)^{-1}\right)^{T}=A\left(q_{0}^{*}\right)^{-T}=\operatorname{sk}\left(q_{0}^{*}\right)+\frac{1}{\lambda} q_{0}^{*} q_{0}^{* T} .
$$

Deriving, $A\left(q_{0}^{*}\right)^{-T}$ one obtains

$$
\begin{aligned}
\frac{d}{d t}\left(A\left(q_{0}^{*}\right)^{-T}\right)= & -\operatorname{sk}\left(\Omega \times q_{0}^{*}\right)-\frac{1}{\lambda} \operatorname{sk}(\Omega) q_{0}^{*} q_{0}^{* T} \\
& +\frac{1}{\lambda} q_{0}^{*} q_{0}^{* T} \operatorname{sk}(\Omega)
\end{aligned}
$$

Using the relation

$$
\operatorname{sk}\left(\Omega \times q_{0}^{*}\right)=\operatorname{sk}(\Omega) \operatorname{sk}\left(q_{0}^{*}\right)-\operatorname{sk}\left(q_{0}^{*}\right) \operatorname{sk}(\Omega)
$$

the derivative of $A\left(q_{0}^{*}\right)^{-T}$ may be rewritten as

$$
\begin{aligned}
\frac{d}{d t}\left(A\left(q_{0}^{*}\right)^{-T}\right)= & -\operatorname{sk}(\Omega) \operatorname{sk}\left(q_{0}^{*}\right)+\operatorname{sk}\left(q_{0}^{*}\right) \operatorname{sk}(\Omega) \\
& -\frac{1}{\lambda} \operatorname{sk}(\Omega) q_{0}^{*} q_{0}^{* T}+\frac{1}{\lambda} q_{0}^{*} q_{0}^{* T} \operatorname{sk}(\Omega) \\
= & -\operatorname{sk}(\Omega) A(q)^{-T}+A(q)^{-T} \operatorname{sk}(\Omega) .
\end{aligned}
$$

Deriving $\delta_{2}$ and recalling (2), (23), and (27), one obtains

$$
\dot{\delta}_{2}=-\operatorname{sk}(\Omega) \delta_{2}+\frac{k_{1}}{m} H \delta_{1}+\frac{k_{1}}{m} H \delta_{2}+\frac{1}{k_{1}} A\left(q_{0}^{*}\right)^{-T} F .
$$

Let $S_{2}$ be a second storage function associated with the translational dynamics

$$
S_{2}=\frac{1}{2}\left|\delta_{1}\right|^{2}+\frac{1}{2}\left|\delta_{2}\right|^{2} .
$$

Taking the time derivative of $S_{2}$, it follows that

$$
\dot{S}_{2}=-\frac{k_{1}}{m} \delta_{1}^{T} H \delta_{1}+\frac{k_{1}}{m} \delta_{2}^{T} H \delta_{2}+\frac{1}{k_{1}} \delta_{2}^{T} A\left(q_{0}^{*}\right)^{-T} F .
$$

The positive-definite matrix $H=A\left(q_{0}^{*}\right) Q A\left(q_{0}^{*}\right)^{T}$ is not exactly known; however, for a suitable choice of $\lambda$, it will be well conditioned with known bounds on eigenvalues in a large neighborhood of the desired set point. Thus, choosing

$$
F:=-\frac{k_{1}^{2} k_{2}}{m} A\left(q_{0}^{*}\right)^{T} \delta_{2}
$$

where $k_{2}>\lambda_{\max }(H)=\max \left\{\lambda_{\max }(Q), \lambda^{2} \lambda_{\min }(Q)\right\}$, is sufficient to stabilize the translational dynamics. Since the rigid body system considered is underactuated, the force input $F$ cannot be directly assigned. The proposed control algorithm continues the backstepping procedure by using the aforementioned definition as a virtual input. A virtual differentiation $\dot{T}$ of thrust is introduced in the following development to ensure decoupling between translational and rotational dynamics, as shown in the sequel (37).

Set

$$
F^{v}:=-\frac{k_{1}^{2} k_{2}}{m} \delta_{2}
$$

A new error term $\delta_{3}$ is defined to measure the scaled difference between the virtual and the true force inputs

$$
\delta_{3}:=\frac{m}{k_{1}^{2} k_{2}} A\left(q_{0}^{*}\right)^{-T} F+\delta_{2} .
$$

The derivative of $\delta_{2}(28)$ becomes

$$
\dot{\delta}_{2}=-\operatorname{sk}(\Omega) \delta_{2}+\frac{k_{1}}{m} H \delta_{1}-\frac{k_{1}}{m}\left(k_{2} I_{3}-H\right) \delta_{2}+\frac{k_{1}}{m} k_{2} \delta_{3}
$$

and the derivative of the second storage function is now

$$
\dot{S}_{2}=-\frac{k_{1}}{m} \delta_{1}^{T} H \delta_{1}-\frac{k_{1}}{m} \delta_{2}^{T}\left(k_{2} I_{3}-H\right) \delta_{2}+\frac{k_{1}}{m} k_{2} \delta_{2}^{T} \delta_{3}
$$

Deriving $\delta_{3}$ and recalling (28), yields

$$
\begin{aligned}
\dot{\delta}_{3}= & -\operatorname{sk}(\Omega) \delta_{3}+\frac{k_{1}}{m} H \delta_{1}-\frac{k_{1}}{m}\left(k_{2} I_{3}-H\right) \delta_{2}+\frac{k_{1}}{m} k_{2} \delta_{3} \\
& +\frac{m}{k_{1}^{2} k_{2}} A\left(q_{0}^{*}\right)^{-T}(\dot{F}+\operatorname{sk}(\Omega) F)
\end{aligned}
$$

Recalling (5), the full vectorial term $(\dot{F}+\operatorname{sk}(\Omega) F)$ is explicitly given by

$$
(\dot{F}+\operatorname{sk}(\Omega) F)=\left(\begin{array}{ccc}
0 & T & 0 \\
-T & 0 & 0 \\
0 & 0 & 1
\end{array}\right)\left(\begin{array}{c}
\Omega_{1} \\
\Omega_{2} \\
\dot{T}
\end{array}\right) .
$$

The goal of the paper is to control the full system dynamics (1)-(4). In practice, the IMU onboard a flying vehicle provides high-bandwidth low-noise measurements of angular velocity $\Omega$ of the vehicle. This allows us to apply a high gain control loop around the angular dynamics (4) and use the angular velocity $\Omega$ as an input to the remainder of the system dynamics (1)-(3). The control of (1)-(3) relies on much lower bandwidth visual feedback and occurs at a much lower bandwidth than the angular velocity control. In fact, only the first two components of the angular velocity $\Omega_{1}$ and $\Omega_{2}$ are required in the visual servo control loop, along with the set point for the dynamic extension of the thrust $\dot{T}$.

Theorem 5.1: Consider the system dynamics (1)-(3) with inputs $\left(\Omega_{1}, \Omega_{2}, \dot{T}\right)$. Let $\delta_{1}$ be defined by (20) and $\delta_{2}, \delta_{3}$ be defined by (24) and (33), respectively. Choose $\left(\Omega_{1}, \Omega_{2}, \dot{T}\right)$ according to (37) such that

$$
\frac{m}{k_{1}^{2} k_{2}}(\dot{F}+\operatorname{sk}(\Omega) F):=-\frac{\left(k_{1} k_{2}+k_{3}\right)}{m} A\left(q_{0}^{*}\right)^{T} \delta_{3}
$$

for $k_{1}, k_{3}>0$ and $k_{2}>\lambda_{\max }(H)$. Then, $\delta_{1}$ is locally exponentially stable to zero and the attitude direction $R^{T} e_{3}$ is locally exponentially stable to $e_{3}$.

Proof: Let $\mathcal{L}$ be a Lyapunov candidate function defined by

$$
\mathcal{L}=\frac{1}{2}\left|\delta_{1}\right|^{2}+\frac{1}{2}\left|\delta_{2}\right|^{2}+\frac{1}{2}\left|\delta_{3}\right|^{2}=S_{2}+\frac{1}{2}\left|\delta_{3}\right|^{2} .
$$

Taking the derivative of $\mathcal{L}$, and recalling (35) and (36), one obtains

$$
\begin{aligned}
\dot{\mathcal{L}}= & -\frac{k_{1}}{m} \delta_{1}^{T} H \delta_{1}-\frac{k_{1}}{m} \delta_{2}^{T}\left(k_{2} I_{3}-H\right) \delta_{2}+\frac{k_{2}}{m} \delta_{2}^{T} \delta_{3} \\
& \times \frac{k_{1}}{m} \delta_{3}^{T} H \delta_{1}-\frac{k_{1}}{m} \delta_{3}^{T}\left(k_{2} I_{3}-H\right) \delta_{2}+\frac{k_{1}}{m} k_{2} \delta_{3}^{T} \delta_{3} \\
& +\frac{m}{k_{1}^{2} k_{2}} \delta_{3}^{T} A\left(q_{0}^{*}\right)^{-T}(\dot{F}+\operatorname{sk}(\Omega) F)
\end{aligned}
$$


Introducing the expression (38) in the Lyapunov function derivative, one obtains

$$
\begin{aligned}
\dot{\mathcal{L}}= & -\frac{k_{1}}{m} \delta_{1}^{T} H \delta_{1}-\frac{k_{1}}{m} \delta_{2}^{T}\left(k_{2} I_{3}-H\right) \delta_{2} \\
& +\frac{k_{1}}{m} \delta_{3}^{T} H \delta_{1}-\frac{k_{1}}{m} \delta_{3}^{T}\left(k_{2} I_{3}-H\right) \delta_{2}-\frac{k_{3}}{m} \delta_{3}^{T} \delta_{3} .
\end{aligned}
$$

Completing the square three times to dominate the cross terms, it may be verified that the choice of the control gains given in the theorem ensures that the right-hand side is negative definite in all the error signals $\delta_{i}, i=1, \ldots, 3$. The classical Lyapunov theory ensures exponential convergence of $\delta_{i} \rightarrow 0$.

If the position and linear velocity are regulated, then the total external force must be zero, $F=0$. Recalling (5) one has

$$
R^{T} e_{3}=e_{3}, \quad T=m g .
$$

Note that the error term $\delta_{3}$ does not determine the full attitude of the system considered. Only pitch and roll components of the attitude are regulated by the error $\delta_{3}$ while the yaw rotation around the thrust direction is independent of the error criteria. In practice, it is desirable to stabilize the yaw of the vehicle to avoid unwanted second-order dynamic effects and provide a stabile platform for sensor systems. Different solutions may be used to stabilize the freedom of yaw rotation in the attitude dynamics. An additional visual error is proposed in Hamel and Mahony [14]; however, this leads to significant additional complexity in the mathematical development. To avoid complexity, a simple damping term

$$
\Gamma_{3}=-k_{4} \Omega_{3}, \quad k_{4}>0
$$

can be used to stop unwanted rotation without specifying a specific yaw set point. The solution adopted in Section VI is a hybrid control, where the position, pitch, and roll of the vehicle are controlled autonomously, while the yaw is manually servocontrolled using the operator joystick.

\section{EXPERIMENTAL RESULTS}

In this section, the control algorithm presented in Proposition 5.1 is implemented on a quadrotor, made by the CEA (Fig. 1).

A quadrotor is a vertical takeoff and landing vehicle ideally suited for stationary and quasi-stationary flight. The vehicle consists of four individual fans fixed to a rigid cross frame. An idealized dynamic model of the quadrotor [1], [17] is given by the rigid body equations (1)-(4) along with the external force and torque inputs (cf.Fig. 2)

$$
\begin{aligned}
T & =T_{r r}+T_{r l}+T_{f r}+T_{f l} \\
\Gamma_{1} & =d\left(T_{f r}+T_{f l}-T_{r r}-T_{r l}\right) \\
\Gamma_{2} & =d\left(T_{r l}+T_{f l}-T_{r r}-T_{f r}\right) \\
\Gamma_{3} & =Q\left(T_{f r}\right)+Q\left(T_{r l}\right)+Q\left(T_{f l}\right)+Q\left(T_{r r}\right) \\
& =\kappa\left(T_{f r}+T_{r l}-T_{f l}-T_{r r}\right) .
\end{aligned}
$$

The individual thrust of each motor is denoted $T_{(.)}$, while $\kappa$ is the proportional constant giving the induced couple due to air
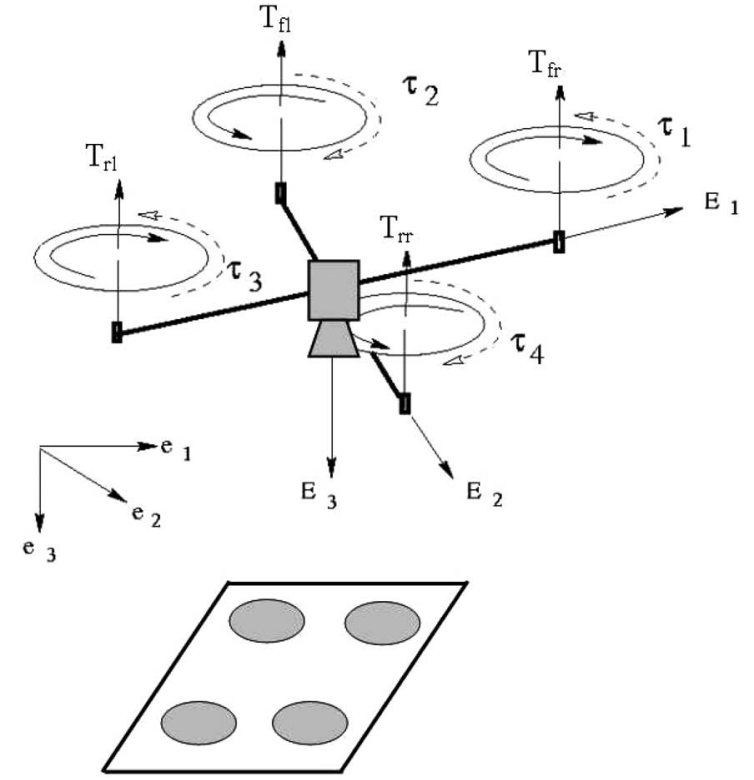

Fig. 2. Force and torque inputs for an X4-flyer.

resistance for each rotor and $d$ denotes the distance of each rotor from the center of mass of the quadrotor.

The control set point for $T$ is obtained by integration of the third component of (38), while the control torques $\Gamma_{1}$ and $\Gamma_{2}$ are obtained via a high-gain stabilization of the first two components of (4) to the control set points given by the first two components of (38). The final torque component $\Gamma_{3}$ is independently determined via high-gain feedback control to a set point $\Omega_{3}$ derived from the joystick.

The parameters used for the dynamic model have been identified as follows: $m=0.55 \mathrm{~kg}, I=\operatorname{diag}(0.009,0.009$, $0.018) \mathrm{kg} \cdot \mathrm{m}^{2}, d=0.23 \mathrm{~m}, \kappa=0.018 \mathrm{~m}$ and $g=9.8 \mathrm{~m} \cdot \mathrm{s}^{-2}$.

\section{A. Prototype Description}

The CEA's quadrotor is equipped with a set of four electronic boards [Fig. 3(b)] designed by their staff. Vibration-absorbent material was placed between the electronic boards and the airframe to minimize sensor noise in the microelectromechanical system (MEMS) sensor components. Each electronic board includes a microcontroller and has a particular function. The first board integrates the motor controllers that regulate the rotation speed of the four propellers. The second board integrates an IMU, developed by the CEA, consisting of three low-cost MEMS accelerometers, three angular rate sensors, and two magnetometers. The explicit complementary filter [16] is used to estimate the attitude vector (pitch and roll) and gyros bias from the IMU data. On the third board, a digital signal processing (DSP), running at 150 MIPS, is embedded and performs the control algorithm and filtering computations. The final board provides a serial wireless communication between the operator's joystick and the vehicle. An embedded camera [Fig. 3(a)] with a field of view of $120^{\circ}$ is mounted pointing down, and transmits video to a ground station personal computer (PC) via a wireless analogical link at $2.4 \mathrm{GHz}$. Finally, a lithium-polymer battery provides 


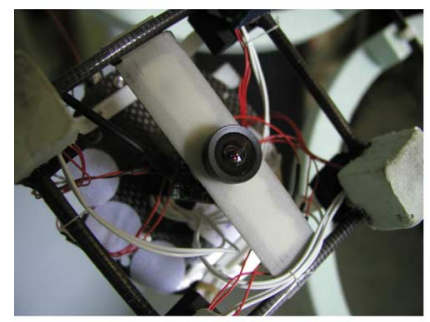

(a)

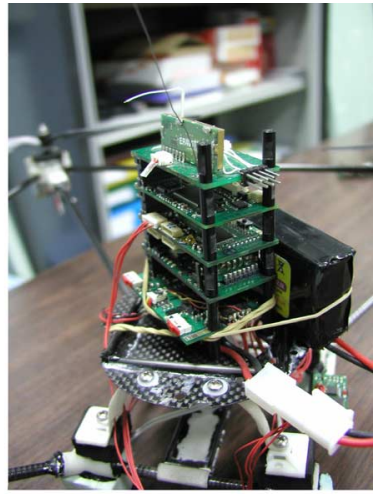

(b)
Fig. 3. (a) Embedded camera. (b) Set of electronic boards.

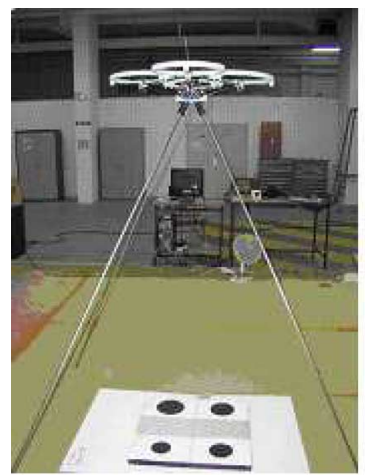

(a)

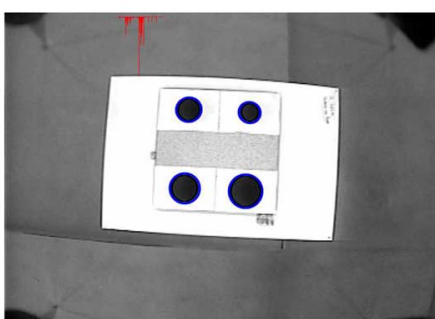

(b)
Fig. 4. (a) Initialization of the algorithm. (b) Target view from the camera.

nearly 10 min of flight time. The images sent by the embedded camera are received by the ground station at a frequency of $15 \mathrm{~Hz}$. In parallel, the quadrotor sends the inertial data to the ground station at $9 \mathrm{~Hz}$. The data is processed by the ground station PC and incorporated into the control algorithm. The visual servo control algorithm is computed in the ground station PC and provides desired orientation velocities and a desired thrust rate. These control set points are transmitted to the drone where the high-gain control of the motor torque is embedded on the DSP running at $166 \mathrm{~Hz}$. This high-gain control ensures stability of the vehicle despite the presence of significant latency incurred in the reception and processing of inertial data and visual features and the transmission of control demand.

\section{B. Experiments}

The target considered consists of the four black marks on the vertices of a stationary planar square [Fig. 4(b)]. A standard computer vision segmentation algorithm extracts the marks from the background and computes the central moment of each mark. The central moments are transformed into unit-norm spherical image plane representation using the camera calibration matrix provided by the manufacturer. The four image points obtained in this manner are summed to compute the unnormalized spherical centroid $q$ (9). The characteristics of the experimental camera ensure that the observed target remains visible if the quadrotor

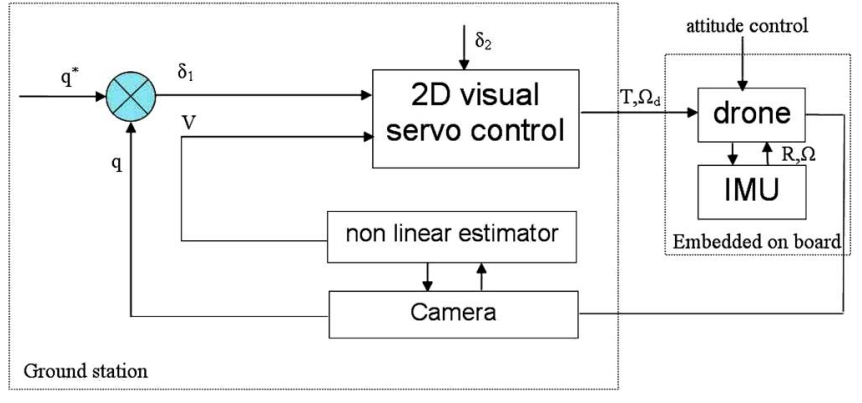

Fig. 5. Schematic block diagram of estimation and control loops.

remains in cone of angle $\approx 45^{\circ}$ around the observed target and has at most $\approx 5^{\circ}$ inclination. This generates a workspace of diameter approximately $1.5 \mathrm{~m}$ around the center of the target at an altitude of $1.4 \mathrm{~m}$. The desired image feature $b^{*}$ is chosen such that the camera set point is located $1.4 \mathrm{~m}$ above the target

$$
b^{*} \simeq\left(\begin{array}{c}
0 \\
0 \\
3.9
\end{array}\right)
$$

Fig. 4(a) shows the unmanned aerial vehicle mounted at the set point during the process of acquiring the set point image for the image error.

1) Initialization: To implement the control algorithm, it is necessary to estimate the parameter $\lambda$ that is integral in improving the conditioning of the Jacobian matrix $Q$ around the desired position. The set point for the experiment was set at $(x, y, z) \simeq(0,0,1.4) \mathrm{m}[$ Fig. 4(a)] leading to a Jacobian matrix

$$
Q^{*} \simeq\left(\begin{array}{ccc}
2.35 & 0 & 0 \\
0 & 2.36 & 0 \\
0 & 0 & 0.056
\end{array}\right)
$$

The condition number of $Q^{*}$ is $\rho\left(Q^{*}\right)=\lambda_{\max }\left(Q^{*}\right) / \lambda_{\min }\left(Q^{*}\right)$ $\simeq 42.14$. The asymptotic convergence rates of the proposed algorithm are given by the eigenvalues of $H=A\left(q_{0}^{*}\right) Q^{*} A\left(q_{0}^{*}\right)^{T}$. For the experimental configuration considered, one has (22)

$$
A\left(q_{0}^{*}\right)=\left(\begin{array}{ccc}
0 & 1 & 0 \\
-1 & 0 & 0 \\
0 & 0 & \lambda
\end{array}\right)
$$

Choosing $\lambda=6.44$, one obtains

$$
A\left(q_{0}^{*}\right) Q^{*} A\left(q_{0}^{*}\right)^{T}=2.35\left(\begin{array}{ccc}
1 & 0 & 0 \\
0 & 1 & 0 \\
0 & 0 & 1
\end{array}\right) .
$$

Since $H=A\left(q_{0}^{*}\right) Q A\left(q_{0}^{*}\right)^{T} \approx A\left(q_{0}^{*}\right) Q^{*} A\left(q_{0}^{*}\right)^{T}$ in the vicinity of the set point, it is expected that the overall system performance will be acceptable.

2) Results: During the experiments, the yaw velocity $\left(\Omega_{3}\right)$ was controlled via the joystick. Yaw velocity does not affect the proposed control scheme (37) and the convergence of the closed-loop system is independent of the operator input. The drone is flown under manual control into the neighbourhood of the target to ensure the target marks are visible before the 

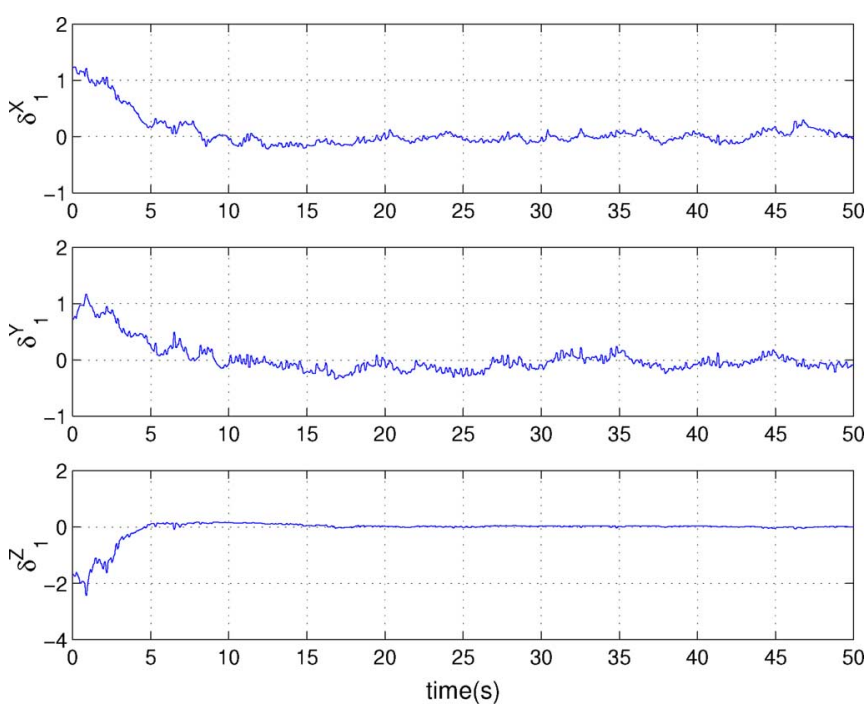

Fig. 6 Error term $\delta_{1}$.
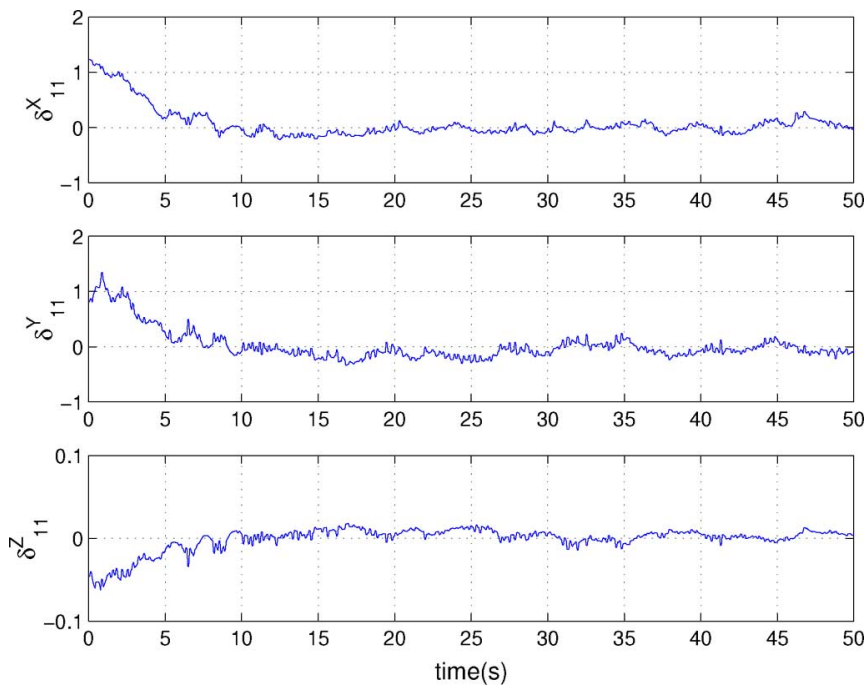

Fig. 7. Error term $\delta_{11}$.

control algorithm is engaged. An estimate of the initial position is $\left(x_{0}, y_{0}, z_{0}\right) \simeq(0.7,-0.8,2) \mathrm{m}$.

The exponential convergence of the visual error $\delta_{1}$ is clearly visible in Fig. 6. The separate convergence of the error terms $\delta_{11}$ and $\delta_{12}$ are shown in Figs. 7 and 8. Fig. 9 shows the evolution of the centroid vector, $q$, and Fig. 10 shows the convergence of the thrust direction $R_{3}$ to $e_{3}$. Fig. 11 shows the evolution of target points in the image space. Fig. 12 shows the position evolution of the quadrotor in the Cartesian space as obtained from the fullpose estimation algorithm that was run separately in parallel to the control algorithm. ${ }^{2}$ The closed-loop performance of the system maintains an error of approximately $10 \mathrm{~cm}$ around the desired position (Fig. 12). The authors believe that the most significant source of error is due to aerodynamic disturbances

${ }^{2}$ Note that the only place where the full-pose estimates are used is in plotting Fig. 12, although the pose estimation algorithm is used to generate the estimate of linear velocity used in the control algorithm.

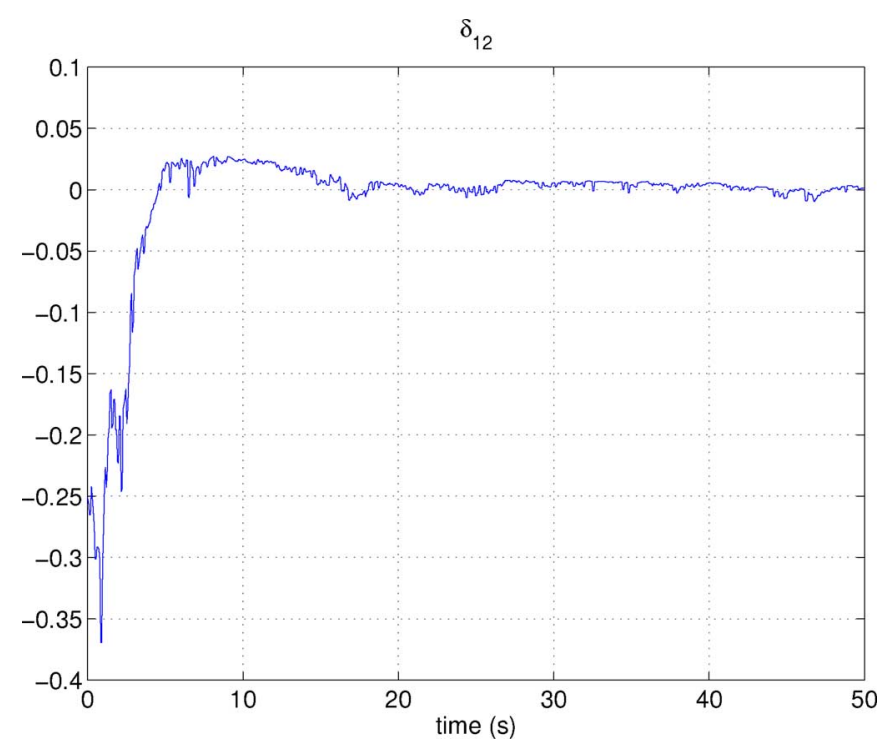

Fig. 8. Error term $\delta_{12}$.
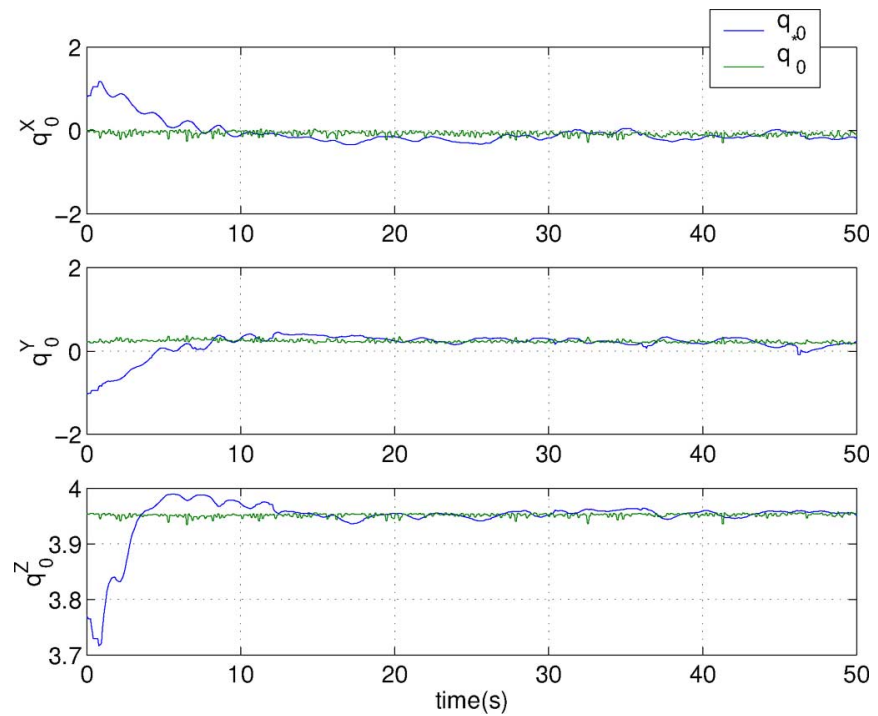

Fig. 9. Centroid evolution.

that can be considered as a load disturbance to the system. Any rotor craft creates vortices at the tips of the rotor plane when in hover. These vortices grow in size and strength, and then become unstable and get sucked through the rotor, causing a momentary loss of lift, before a new vortex begins to grow. Interestingly, this effect is worst in stationary hover conditions as translation through the air causes the protovortices to be washed through the rotor before they have built-up energy. Other sources of error in the closed-loop system may come from system modeling and transmission delays. Despite the errors, experiments show that the regulation error remains bounded and smaller than $10 \mathrm{~cm}$ around the desired position. The authors feel that the practical stability is very good with regards to the experimental system considered. 

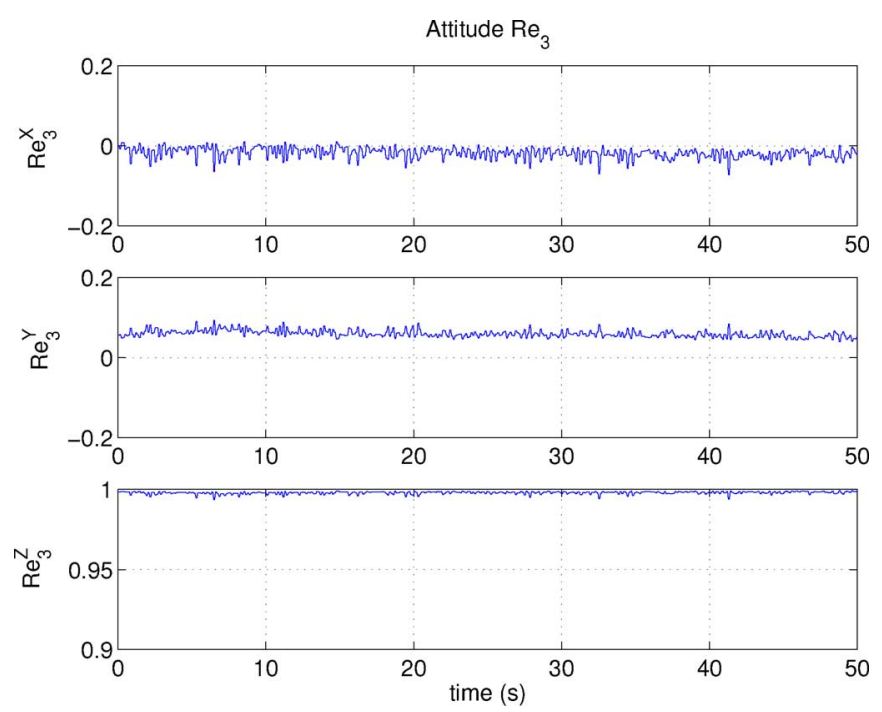

Fig. 10. Evolution of the components of the vector $\mathrm{Re}_{3}$.

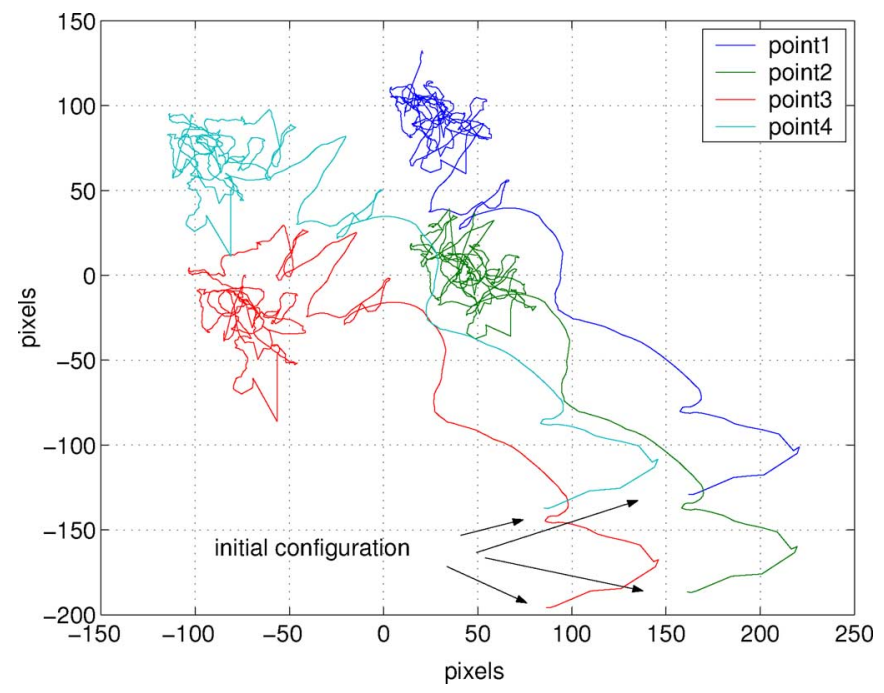

Fig. 11. Trajectory in the image plan of the four black marks.

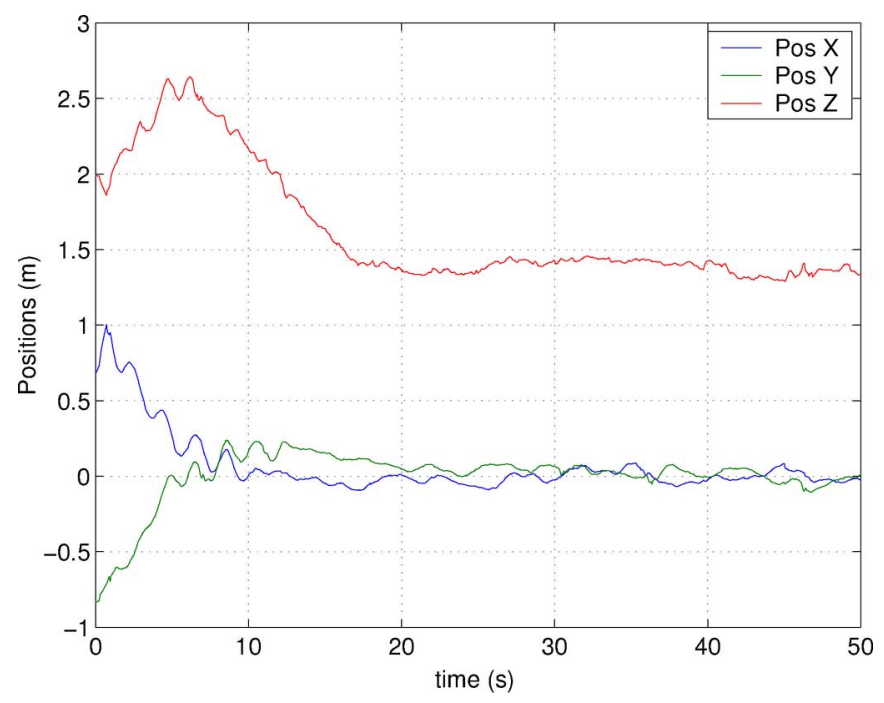

Fig. 12. Three-dimensional UAV position.

\section{CONCLUSION}

In this paper, we presented a visual servo control for stabilization of a quadrotor UAV. This research is an extension of the recent theoretical work on visual servo control of underactuated systems [14] that overcomes ill-conditioning of the Jacobian matrix. Based on the previous research [4], a new visual error is proposed that improves the conditioning of the closed-loop Jacobian matrix in the neighborhood of the desired set point. A nonlinear controller is derived, using backstepping techniques, and implemented on an experimental flying robot developed by the CEA. The experimental results show good performance and robustness of the proposed control strategy.

\section{REFERENCES}

[1] E. Altug, J. Ostrowski, and R. Mahony, "Control of a quadrotor helicopter using visual feedback," in Proc. IEEE Int. Conf. Robot. Autom., ICRA, 2002, vol. 1, pp. 72-77.

[2] R. L. Andersson, A Robot Ping-Pong Player: Experiment in Real-Time Intelligent Control. Cambridge, MA: MIT Press, 1988.

[3] A. Astolfi, L. Hsu, M. Netto, and R. Ortega, "Two solutions to the adaptive visual servoing problem," IEEE Trans. Robot. Autom., vol. 18, no. 3, pp. 387-392, Jun. 2002.

[4] O. Bourquardez, R. Mahony, T. Hamel, and F. Chaumette, "Stability and performance of image based visual servo control using first order spherical image moments," in Proc. IEEE/RSJ Int. Conf. Intell. Robots Syst. IROS 2006, Beijing, China, Oct., pp. 4304-4309.

[5] A. Castano and S. Hutchinson, "Visual compliance: Task directed visual servo control," IEEE Trans. Robot. Autom., vol. 10, no. 3, pp. 334-341, Jun. 1993.

[6] F. Chaumette, "Potential problems of stability and convergence in imagebased and position-based visual servoing," in Proc. Conf. Vis. Control, LNCIS, 1998, vol. 237, pp. 66-78.

[7] T. Chevirond, T. Hamel, R. Mahony, and G. Baldwin, "Robust nonlinear fusion of inertial and visual data for position, velocity and attitude estimation of a UAV," in Proc. IEEE Int. Conf. Robot. Autom. ICRA 2007, Roma, Italy, Apr. 10-14, pp. 2010-2016.

[8] P. I. Corke and S. A. Hutchinson, "A new partitioned approach to imagebased visual servo control," presented at the Int. Symp. Robot., Montreal, QC, Canada, May 2000.

[9] P. I. Corke and S. A. Hutchinson, "A new hybrid image-based visual servo control scheme," in Proc. IEEE Int. Conf. Decis. Control, CDC 2000, Sydney, Australia, Dec., pp. 2521-2526.

[10] K. Deguchi, "Optimal motion control for image-based visual servoing by decoupling translation and rotation," in Proc. Int. Conf. Intell. Robots Syst., 1998, pp. 705-711.

[11] B. Espiau, F. Chaumette, and P. Rives, "A new approach to visual servoing in robotics," IEEE Trans. Robot. Autom., vol. 8, no. 3, pp. 313-326, Jun. 1992.

[12] C. Fermuller and Y. Aloimonos, "Observability of 3D motion," Int. J. Comput. Vis., vol. 37, no. 1, pp. 43-64, Jun. 2000.

[13] E. Frazzoli, M. A. Dahleh, and E. Feron, "Real-time motion planning for agile autonomous vehicles," AIAA J. Guid. Control, Dyn., vol. 5, no. 1, pp. 116-129, 2002.

[14] T. Hamel and R. Mahony, "Visual servoing of an under-actuated dynamic rigid-body system: An image based approach," IEEE Trans. Robot. Autom., vol. 18, no. 2, pp. 187-198, Apr. 2002.

[15] T. Hamel and R. Mahony, "Image based visual servo-control for a class of aerial robotic systems," Automatica, vol. 43, pp. 1975-1983, 2007.

[16] T. Hamel and R. Mahony, "Attitude estimation on $S O(3)$ based on direct inertial measurements," in Proc. Int. Conf. Robot. Autom. ICRA 2006, Orlando, FL, May 15-19, pp. 2170-2175.

[17] T. Hamel, R. Mahony, R. Lozano, and J. Ostrowski, "Dynamic modelling and configuration stabilization for an X4-flyer," presented into to Int. Fed. Automat. Control Symp., IFAC 2002, Barcelona, Spain.

[18] S. Hutchinson, G. Hager, and P. Cork, "A tutorial on visual servo control," IEEE Trans. Robot. Autom., vol. 12, no. 5, pp. 651-670, Oct. 1996.

[19] K. P. Khosla, N. Papanikolopoulos, and B. Nelson, "Dynamic sensor placement using controlled active vision," in Proc. IFAC 12th World Congr. Sydney, Australia, 1993, pp. 9.419-9.422. 
[20] M. Lei and B. K. Ghosh, "Visually guided robotic motion tracking," in Proc. 13th Annu. Conf.Commn., Control Comput., 1992, pp. 712-721.

[21] R. Mahony, P. Corke, and T. Hamel, "Dynamic image-based visual servo control using centroid and optic flow features," J. Dyn. Syst. Meas. Control, vol. 130, no. 1, pp. 011005-1-011005-12, Jan. 2008.

[22] R. Mahony and T. Hamel, "Robust trajectory tracking for a scale model autonomous helicopter," Int. J. Non-linear Robust Control, vol. 14, pp. 1035-1059, 2004.

[23] R. Mahony, T. Hamel, and F. Chaumette, "A decoupled image space approach to visual servo control of a robotic manipulator," in Proc. IEEE Int. Conf. Robot. Autom. ICRA 2002, Washington, DC, May., pp. 37813786.

[24] E. Malis, F. Chaumette, and S. Boudet, "2-1/2-D visual servoing," IEEE Trans. Robot. Autom., vol. 15, no. 2, pp. 238-250, Apr. 1999.

[25] N. Metni and T. Hamel, "Visual tracking control of aerial robotic systems with adaptive depth estimation," Int.J. Control Autom., Syst., vol. 5, no. 1, pp. 51-60, 2007.

[26] L Mejias, S Saripalli, G. S Sukhatme, and P Cervera, "Visual servoing for tracking features in urban areas using an autonomous helicopter," J. Field Robot., vol. 23, no. 3-4, pp. 185-199, 2006.

[27] G. Morel, T. Liebezeit, J. Szewczyk, S. Boudet, and J. Pot, "Explicit incoporation of 2D constraints in vision based control of robot manipulators," in Lecture Notes in Control and Information Sciences, vol. 250, P. Corke and J. Trevelyan, Eds. New York: Springer-Verlag, 1999, pp. 99108.

[28] N. Papanikolopoulos, P. K. Khosla, and T. Kanade, "Adaptive robot visual tracking," in Proc. Amer. Control Conf., 1991, pp. 962-967.

[29] J. A. Piepmeier, "A dynamic quasi-newton method for model independent visual servoing," Ph.D. dissertation, Georgia Inst.Technol., Atlanta, GA, Jul. 1999.

[30] R. Pissard-Gibollet and P. Rives, "Applying visual servoing techniques to control of a mobile hand-eye system," in Proc. IEEE Int. Conf. Robot. Autom., ICRA 1995, Nagasaki, Japan, pp. 166-171.

[31] O. Shakernia, Y. Ma, T.J. Koo, and S. Sastry, "Landing an unmanned air vehicle: Vision based motion estimation and nonlinear control," Asian J. Control, vol. 1, no. 3, pp. 128-146, 1999.

[32] H Shim, T. Koo, F Hoffmann, and S Sastry, "A comprehensive study of control design for an autonomous helicopter," in Proc. 37th Conf. Decision Control, Tampa, FL, 1998, pp. 3653-3658.

[33] B Yoshimi and P.K. Allen, "Active, uncalibrated visual servoing," in Proc. IEEE Int. Conf. Robot. Autom. ICRA 1994, San Diago, CA, 1994, pp. 156 161.

[34] E. Zergeroglu, D. Dawson, M. de Queiroz , and S. Nagarkatti, "Robust visual-servo control of robot manipulators in the presence of uncertainty," in Proc. 38th Conf. Decision Control, 1999, pp. 4137-4142.

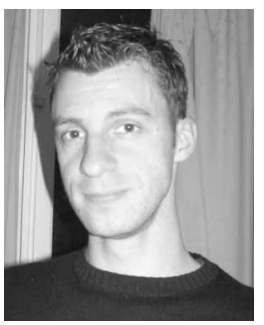

Nicolas Guenard received the Graduate degree from the East Texas Area Claims Association(ETACA), French Engineering School in Automobile, Aeronautics, and Aerospace, Toulouse, France, in 2003, and the Ph.D. degree in sciences from Nice-Sophia Antipolis University, Nice, France, in 2007.

His current research interests include localization and navigation of unmanned aerial vehicles.

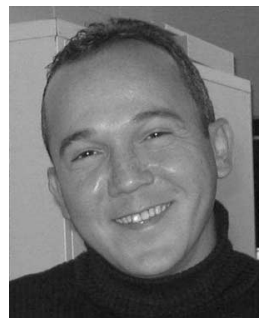

Tarek Hamel (M'07) received the Bachelor's degree in engineering from the University of Annaba, Annaba, Algeria, in 1991, and the Ph.D. degree in robotics from the University of Technology Compiègne (UTC), Compiegne, France, in 1995.

$\mathrm{He}$ was a Research Assistant at the UTC for 2 years, and later, became an Associate Professor in the Centre d'Etudes de Mècanique d'Iles de France in 1997. Since 2003, he has been a Professor at the Laboratoire I3S, University of Nice-Sophia Antipolis-National Centre for Scientific Research (UNSA-CNRS), Nice, France. His current research interests include nonlinear control theory, estimation, and vision-based control with applications to unmanned aerial vehicles and mobile robots.

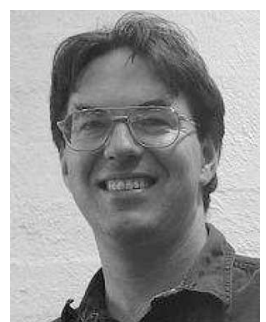

Robert Mahony (M'92-SM'08) received the B.Sc. degree in applied mathematics and geology and the Ph.D. degree in systems engineering from the Australian National University, Canberra, Australia, in 1989 and 1995, respectively.

He was a Marine Seismic Geophysicist and an Industrial Research Scientist before completing a 2-year postdoctoral fellowship in France and a 2-year Logan fellowship at Monash University, Australia. Since 2001, he is a Reader in the Department of Engineering, Australian National University, Canberra, A.C.T., Australia. His current research interests include nonlinear control theory with applications in robotics, geometric optimization techniques, and learning theory. 